\title{
ESR teleradiology survey: results
}

\author{
European Society of Radiology (ESR)
}

Received: 11 March 2016 / Accepted: 11 March 2016/Published online: 17 May 2016

(C) The Author(s) 2016. This article is published with open access at Springerlink.com

\begin{abstract}
With recent developments of teleradiology technology and services, it has become necessary to better evaluate its extent and use among different countries in Europe. With this goal in mind, the ESR launched two specific surveys intended to gather the current state of adoption and implementation of teleradiology in clinical practice. A special focus on differentiating between insourcing teleradiology services among partners of the same organisation and outsourcing to external services was an essential part of the design of these surveys. The first survey was addressed to 44 national societies of different countries in Europe, while the second survey was intended for all practicing radiologist ESR members. While the results of these surveys reported here may provide a wealth of information to better understand the trends in adoption of teleradiology in Europe, they only represent a snapshot at a certain point in time. The rapid development of telecommunication tools as well as a fundamental change in practice and healthcare economics will certainly influence these observations in the upcoming years. These data, however, will provide objective and relevant parameters for supporting the efforts of experts
\end{abstract}

European Society of Radiology (ESR)

communications@myesr.org

1 Neutorgasse 9/2, 1010 Vienna, Austria and policy makers in promoting appropriate criteria and guidelines for adequate use of teleradiology in clinical practice.

Main Messages

- Understand concepts and challenges of teleradiology

- Provide insight into current trends and solutions for teleradiology

- Compare differences in teleradiolgy strategies between countries in Europe

- Establish a reference on statistical data of usage of teleradiology in Europe

Keywords Teleradiology $\cdot$ PACS $\cdot$ Digital imaging $\cdot$ Imaging infomatics $\cdot$ Remote consultations

\section{Introduction}

The wider adoption of picture archiving and communication systems (PACS) has had significant benefits, including the ability to report on imaging procedures remotely, from the location where they have been performed via teleradiology. With the development of new technical infrastructures and networks allowing faster transfer of images and more accessible workstation designs, the rate of adoption of teleradiology in clinical practice has gained momentum in recent years. However, following publication of the White Paper on Teleradiology by the ESR in Insights into Imaging in 
2014 [1], it has become apparent that the perspective of different National Societies varies, and in some countries there is a significant level of concern about how teleradiology is used currently, or might be used in the future. This particularly relates to outsourcing, and the effects that this might have on the quality and delivery of radiological services.

In order to further explore these differences and widen the scope of new applications of teleradiology and how they are progressively penetrating the market, the ESR eHealth \& Informatics Subcommittee in cooperation with the ESR Quality, Safety and Standards Committee have elected to initiate two new surveys on this topic, one being destined for national societies and the other one destined for practicing radiologists and general members of ESR.

This paper reports the detailed results of these surveys with the aim to provide a global view of the situation of teleradiology in clinical practice in Europe at this point in time. Although it is certainly not comprehensive, it provides sufficient data for a better perception of the impact of different teleradiology strategies and the users opinion as well as the national societies' positions on these emerging and often disruptive changes in practice. Furthermore, the survey sent to the ESR National Societies was designed to try to capture the perspective of National Society members of the ESR on these important issues to help inform the ESR and its future policy.

\section{Methods}

The surveys were performed online and invitations to participate were sent by the ESR office to the ESR National Societies for the first one (referred as the "first" survey) and to the ESR members for the second one (referred as the "second" survey in this paper). Online questionnaires (SurveyMonkey Inc.) were designed and submitted as a web link allowing users to fill in their responses on any internet browser.

The survey submitted to the National societies consisted of 11 questions as well as a section for free text comments (see below in the results section for detailed listing of the questions). The questions were specifically focused on the different impact of insourcing versus outsourcing teleradiology services.

The second survey submitted to full member ESR radiologists consisted of an extended set of 34 questions divided into four sections:

- brief introduction and demographic questions

- information on the impact and user dimension of teleradiology services requested (e.g., in- and outsourcing, dis-/advantages, quality assurance, etc.)

- information on the financial aspects, informed consent as well as guidelines and legislation in the respective field

- evaluation of the survey itself and teleradiology in general

In addition to the specific questions, the survey also included a section of free text for comments and opinion responses.

The data were first extracted and summarised by ESR staff and reviewed by the chair of ESR eHealth \& Informatics Subcommittee and the chair of the ESR Quality, Safety and Standards Committee before submission of the manuscript to the members of the eHealth \& Informatics Subcommittee for review.

\section{Keywords and definitions used in the survey}

Teleradiology Transfer of radiological images and patient-related data between geographically different locations for the purposes of primary interpretation, expert consultation and/or clinical review by digital transmission. (ref. ESR white Paper on teleradiology)

Insourcing Shared or Network reporting - transfer of images between sites to enable the radiologist to work offsite or report images from remote locations, but employment arrangements are unaffected and radiologists are paid by one of the institutions 
Outsourcing Worklists are outsourced to teleradiology companies, which employ radiologists (see more detailed definition in the ESR white

Paper on teleradiology)

\section{(1) Survey to the ESR National Societies}

\section{Results}

25 out of the 44 National Societies invited to participate responded to the survey and the results are tabulated below.

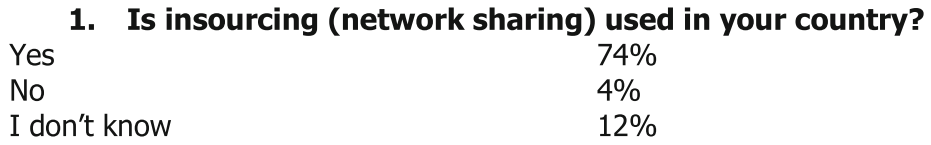

\section{Frequency of insourcing (network, sharing)} Never

$4 \%$

Occasionally (less than $5 \%$ of reports) $\quad 56 \%$

Some of the time (up to $25 \%$ of reports) $\quad 32 \%$

Often (approx. $50 \%$ of reports)

$0 \%$

Very frequently (more than $50 \%$ of reports) $0 \%$

I don't know

$8 \%$

3. When is it usually used? (May indicate more than one of the below)

$\begin{array}{ll}\text { As part of normal daytime service } & 44 \% \\ \text { For out of hours reporting (nighthawk) } & 52 \% \\ \text { For reporting backlogs } & 16 \% \\ \text { For second or specialist opinions } & 56 \% \\ \text { For multidisciplinary decision meetings } & 44 \% \\ \text { I don't know } & 8 \%\end{array}$

4. Is there geographical variation within your country on the use of insourcing i.e some places use it frequently and some not at all?

Yes

$56 \%$

No

$44 \%$

5. Has insourcing (the ability to transfer images) generally had a positive impact on the service?

$\begin{array}{ll}\text { Yes } & 60 \% \\ \text { No } & 12 \% \\ \text { I don't know } & 24 \% \\ \text { Not applicable } & 4 \%\end{array}$

6. Is outsourcing used in your country? Yes

$70.8 \%$

No

$16.7 \%$

I don't know

$12.5 \%$

\section{Frequency of outsourcing}

Never

$16.7 \%$

Occasionally (less than $5 \%$ of reports)

$41.7 \%$

Some of the time (up to $25 \%$ of reports) $\quad 20.8 \%$

Often (approx. $50 \%$ of reports)

$4.2 \%$ 
Very frequently (more than $50 \%$ of reports) $0 \%$

I don't know $16.7 \%$

8. When is it usually used (May indicate more than one of the below)

As part of normal daytime service $\quad 41.7 \%$

For out of hours reporting (nighthawk) $\quad 50 \%$

For reporting backlogs $\quad 29.2 \%$

For second or specialist opinions $\quad 37.5 \%$

I don't know 29.2\%

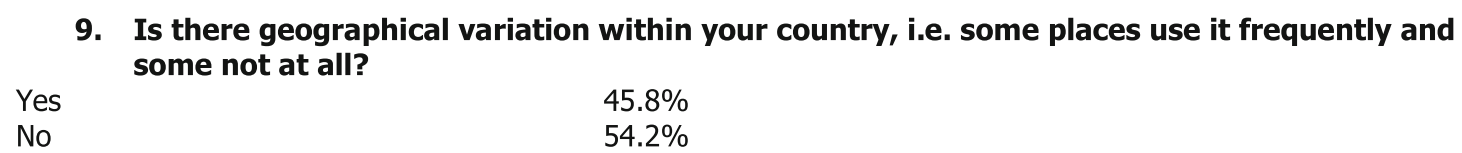

$\begin{array}{lc}\text { 10. Has outsourcing generally had a positive impact on the service? } \\ \text { Yes } & 16.7 \% \\ \text { No } & 33.3 \% \\ \text { I don't know } & 29.2 \% \\ \text { Not applicable } & 20.8 \%\end{array}$

11. Is outsourcing generally supported or not supported by your professional organisation? Supported $29.2 \%$

Not supported $\quad 70.8 \%$

Comparison of insourcing and outsourcing (key issues)

1. Used?

Insourcing Outsourcing

$74 \%$

$56 \%$

$70.8 \%$

2. Less than $5 \%$ of reports

$32 \%$

$41.7 \%$

3. Up to $25 \%$ of reports

$44 \%$

$20.8 \%$

4. Used as Part of daytime service

$52 \%$

$41.7 \%$

5. Nighthawk

$50 \%$

6. Positive effect on service?

$60 \%$

$16.7 \%$

7. Not had a positive effect

$12 \%$

$33.3 \%$

\section{Discussion}

This survey mainly focused on differences in adoption of teleradiology and recommendations of different national societies regarding insourcing and outsourcing of services. However one of the key issues in discussing teleradiology is separating the provision of offsite services from the funding arrangements, and that is quite difficult to capture in a questionnaire. Also, various levels of service, from exam justification to interpretation and reporting may be provided offsite, although it is the remote interpretation and reporting that are most often provided via teleradiology. The general summaries of the survey are listed below.

\section{Insourcing}

The majority of the respondents (74\%) answered that insourcing is used in their country, whereas $4 \%$ answered that insourcing is not used; $12 \%$ answered that they do not know. With regard to the frequency of insourcing (network, sharing), somewhat above half of the respondents $(56 \%)$ indicated that insourcing is used occasionally, i.e., in less than $5 \%$ of the reports. In 
addition, $32 \%$ answered that it is used some of the time, meaning in about up to $25 \%$ of the reports. With respect to the purpose of using insourcing, the respondents could indicate more than one of the various answers offered. The majority $(56 \%)$ indicated that it is mostly used for second or specialist opinions and (52\%) for out of hours reporting nighthawk. The same percentage (44\%) was indicated for multidisciplinary decision meetings and as part of normal daytime service.

In response to the question with regard to geographical variation within a country on the use of insourcing, i.e., some places use it frequently and some not at all; $56 \%$ replied that there indeed is geographical variation, whereas $44 \%$ answered that there is no variation. Free text comments indicated that the use of insourcing is dependent on the hospital organisation and structure. It was also indicated that it is usually used in distant (rural) facilities, smaller hospitals, and some private practices.

Furthermore, $60 \%$ are of the opinion that insourcing (the ability to transfer images) generally had a positive impact on the service. Also, $12 \%$ said that it did not have a positive impact, whereas $24 \%$ answered that they do not know. The respondents could comment on the benefits and problems associated with insourcing (network or sharing). The societies indicated the possibility of receiving a second opinion and consultation in difficult cases and in facilities with limited experience, easier access to images and patient's history, and bypassing the shortage of radiologists and thus managing the workload as the benefits of insourcing. Concerns about reducing the individual radiologist's productivity, the question of remuneration for insourcing, increasing workload in facilities with no specialists, and not perceiving radiologists as doctors were listed as disadvantages of insourcing.

\section{Outsourcing}

For outsourcing, $70.8 \%$ responded that outsourcing is used in their country, whilst in $16.7 \%$ it is not used. Also, $12.5 \%$ of the respondents indicated that they do not know. As regards the frequency of using outsourcing, $41.7 \%$ use it occasionally, i.e., in less than $5 \%$ of the reports and $20.8 \%$ use it in up to $25 \%$ of the reports. With regard to the purpose of using outsourcing, the respondents could again indicate more than one of the various answers offered. Outsourcing is mainly used for out of hours reporting - nighthawk (50\%). It is also used as part of normal daytime service $(41.7 \%)$, for second or specialist opinions $(37.5 \%)$, and for reporting backlogs $(29.2$ $\%)$.

As for the geographical variation within a country on the use of outsourcing, i.e., some places use it frequently and some not at all; $54.2 \%$ answered that there is no variation, whereas $45.8 \%$ said that there is variation. Free text comments indicated that the use of outsourcing is dependent on the network capacity of the hospitals, and that it is mainly used in smaller hospitals. Cross border outsourcing was not addressed in this survey.

Concerning the impact on the service, slightly above one third $(33.3 \%)$ indicated that outsourcing has no positive impact and only $16.7 \%$ responded that outsourcing has a positive impact on the service. There was $29.2 \%$ that answered with "I don't know" and $20.8 \%$ chose the answer "not applicable".

The societies were solicited to comment on the benefits and potential problems associated with outsourcing. Regarding the benefits, respondents indicated the improved turnaround time and less workload, as well as being able to avoid a patient transfer with the help of an expert opinion on an imaging exam. The following are regarded as problems associated with outsourcing: lack of knowledge of the patient data and local circumstances; licensing, accreditation and legislation issues; the question of quality, and follow-up.

\section{Conclusions}

The survey revealed that insourcing and outsourcing are used in many of the National Societies' countries and their use is relatively evenly balanced, insourcing being used slightly more frequently than outsourcing. However when it comes to the positive impact on the service, it is perceived that insourcing mainly has a positive impact (60\%), whereas for outsourcing only $16.7 \%$ perceived the impact to be positive. In $70.8 \%$ of cases, the professional organisation stated that they did not in general support outsourcing. Despite positive effects on workload and quicker turnaround times, outsourcing in particular is associated with significant concerns such as quality, legal issues, and reducing the clinical role of radiologists. 


\section{(2) Survey to the ESR Full Radiologist members}

\section{Results}

568 individual ESR full radiologist members responded to the survey and the results are tabulated below.

\section{Demographic questions (questions 1\&2)}

Participants residing in 26 EU MS ( $¥$ LUX, Malta) participated in the survey. Participants residing in the following non-EU countries: Albania, Belarus, Iceland, Kosovo, Montenegro, Norway, Russian Federation, Serbia, Switzerland, Turkey, Ukraine (other non-European: Aruba, Australia, Israel, Kyrgyzstan, Saudi Arabia, Ukraine, USA) participated in the survey. Participants main activities in 25 EU MS ( $¥$ Cyprus, LUX, Malta).

\section{What is your main professional activity / function?*}

Radiologist in academic hospital Radiologist in hospital with employee status Radiologist both in hospital and private practice (mix) Radiologist in private diagnostic centre, radiology practice Radiologist part-time working for teleradiology company Radiologist in hospital with free entrepreneur status Resident in training (trainee)

Other (please specify)

Radiologist full-time working for teleradiology company Radiologist only active in scientific research

$*$ multiple answers possible

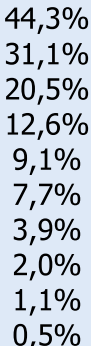

$34,3 \%$

$20,5 \%$

$12,6 \%$

$9,1 \%$

$7,7 \%$

$3,9 \%$

$2,0 \%$

$0,5 \%$

\section{Does your organisation use any type of teleradiology (insourcing or outsourcing)?}
No
$31,7 \%$
Yes
$68,3 \%$

\section{Does your organisation use teleradiology outsourcing?*}

No

Yes, we outsource readings from our backlog (due to capacity problems)

Yes, we outsource on-call readings (nights and weekends)

$27,6 \%$

Yes, we outsource specialized readings (due to lack of expertise)

$23,4 \%$

Other (please specify)

$6,8 \%$

$3,9 \%$

$*$ multiple answers possible

\section{Are radiologists in your organisation involved in the decision making about} outsourcing?

$\begin{array}{lc}\text { Yes, radiologists and management work together in decisions about outsourcing } & 49,2 \% \\ \text { No, decisions to outsource by teleradiology are made by the management } & 30,6 \% \\ \text { Yes, radiologists decide themselves about when and how to outsource } & 13,7 \% \\ \text { I don't know } & 4,4 \% \\ \text { Other (please specify) } & 2,2 \%\end{array}$

7. Does your organisation use teleradiology insourcing?*

$\begin{array}{lc}\text { Yes, work lists are being shared between public hospitals in a regional / area network } & 32,5 \% \\ \text { Yes, for expert / subspecialty readings } & 25,6 \% \\ \text { No insourcing } & 23,4 \% \\ \text { Yes, for on-call purposes (larger regional public hospitals are helping smaller } & 21,5 \% \\ \text { hospitals) } & 13,8 \% \\ \text { Yes, work lists are being shared between private hospitals within the same private } & 8,5 \% \\ \text { hospital group } & 3,6 \% \\ \text { No outsourcing nor insourcing } & \\ \text { I don't know } & \end{array}$


* multiple answers possible

\section{Is teleradiology used for any other purposes in your organisation?*}

No

For providing expert opinions to colleagues (business-to-business model)

$41,6 \%$

For teaching purposes

$40,2 \%$

$15,4 \%$

For scientific research

$12,1 \%$

I don't know

$8,3 \%$

For providing expert opinions directly to patients (business-to-customer model) $\quad 6,1 \%$

We don't use teleradiology at all

$0,6 \%$

* multiple answers possible

\section{Where is the teleradiology workstation located and what kind of workstation is it?*}

on a workstation in our hospital / in our department

$44,1 \%$

on a workstation located in the office of the teleradiology company

$14,8 \%$

on a combination of workstation, mobile devices, own PC/laptop

$14,5 \%$

at home on a professional workstation with equivalent performance to hospital PACS

equipment (not laptop or home PC or Mac)

$12,7 \%$

at home on my own computer (laptop, PC or other)

$11,5 \%$

on a private mobile device (smartphone or tablet)

$1,2 \%$

on a hospital-owned mobile device

$1,2 \%$

* multiple answers possible

\section{How are the teleradiology reports communicated?*}

Teleradiology reports are automatically incorporated in our PACS/RIS system

$78,9 \%$

Offsite radiologist communicates by phone, normal e-mail or other online technique

(VOIP)

Offsite radiologist communicates by secured e-mail (e.g. DICOM e-mail) or other

secured communication tool

$17,8 \%$

Offsite radiologist sends a report by fax

$12,4 \%$

Other (please specify)

$8,8 \%$

$4,5 \%$

Offsite radiologist sends SMS

$0,3 \%$

* multiple answers possible

\section{Can the results be discussed with the offsite radiologist (teleradiologist)?*}

Offsite radiologist can be contacted by phone on a $24 / 7$ basis

$53,8 \%$

Not possible

$16,3 \%$

I don't know

$15,7 \%$

Offsite radiologist can participate in multidisciplinary meetings from distance using a

secure online platform

$10,6 \%$

Offsite radiologist brings visits to our hospital on a regular basis (e.g. for

multidisciplinary meetings)

$9,1 \%$

Offsite radiologist is available for video-consultation (e.g. Skype, Go To Meeting,

other software)

$7,9 \%$

* multiple answers possible

\section{Who can contact the offsite radiologist?*}

Referring clinician (or his/her physician assistant/resident in training) 
I don't know

* multiple answers possible

13. Does the offsite radiologist have access to prior imaging results and/or patient information?

$\mathrm{He} /$ she has access to the PACS/RIS and is able to view prior studies and results

$48,9 \%$

$\mathrm{He} /$ she has access to all relevant patient information including electronic patient record and PACS/RIS

$\mathrm{He} /$ she has no access to our PACS/RIS, prior studies cannot be viewed from

elsewhere

Other (please specify)

I don't know

14. Does your organisation use any quality assurance system for teleradiology?

Yes

No

I don't know

$46,8 \%$

$32,6 \%$

$20,6 \%$

15. What type of quality assurance system is being used?*

I don't know

$36,3 \%$

Offsite radiologists have to be registered in our country and need to follow the national guidelines for local accreditation

$26,8 \%$

Double readings, before the final report is sent

$16,3 \%$

Ad random double readings by the local radiologists

$14,5 \%$

Offsite radiologist or teleradiology company needs formal approval of the medical staff / directors

$13,5 \%$

Auditing of the teleradiology services takes place on a regular basis

$12,9 \%$

Other (please specify)

$9,8 \%$

* multiple answers possible

16. Do you think that the referring doctors in your working environment are satisfied with the teleradiology services?

Yes

No

I don't know
$62,7 \%$

$18,8 \%$

$18,5 \%$ 
17. What do you think are the advantages of using teleradiology in your working environment?*

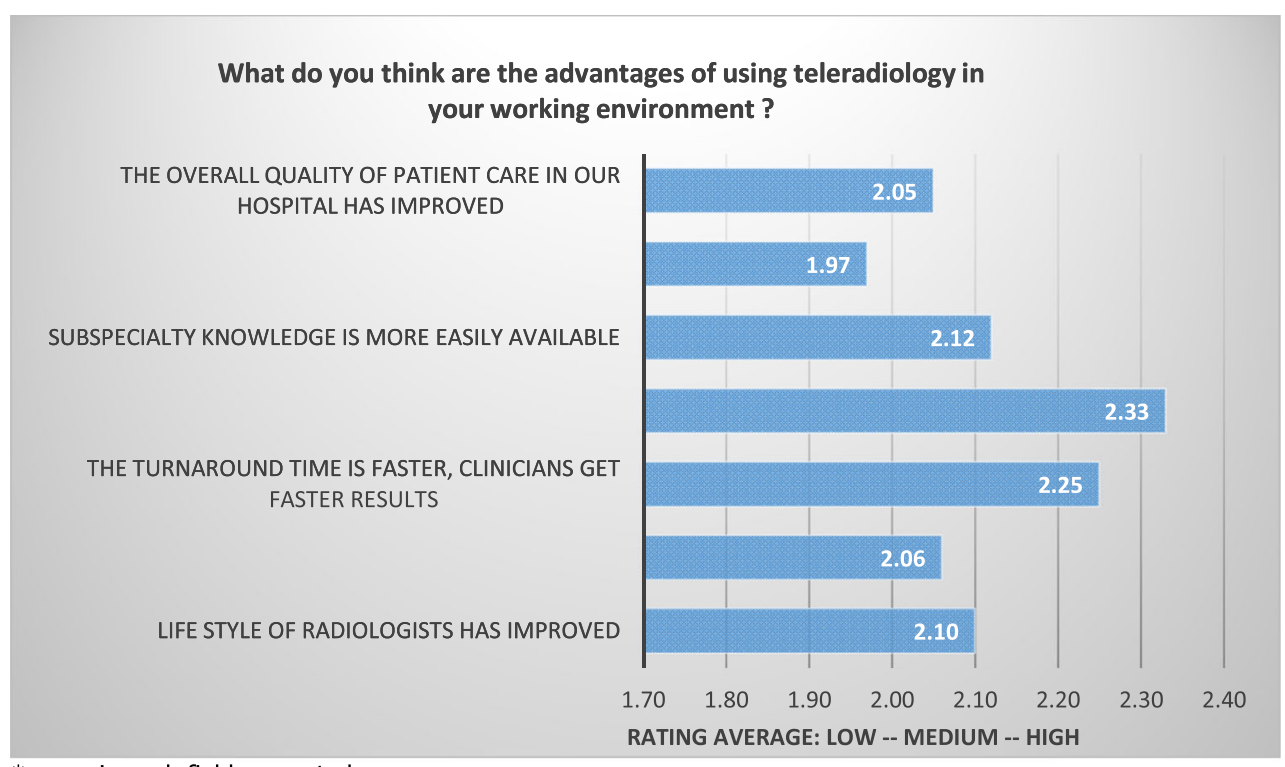

* score in each field requested

18. What do you think are the disadvantages of using teleradiology in your working environment?*

What do you think are the disadvantages of using teleradiology in your working environment?

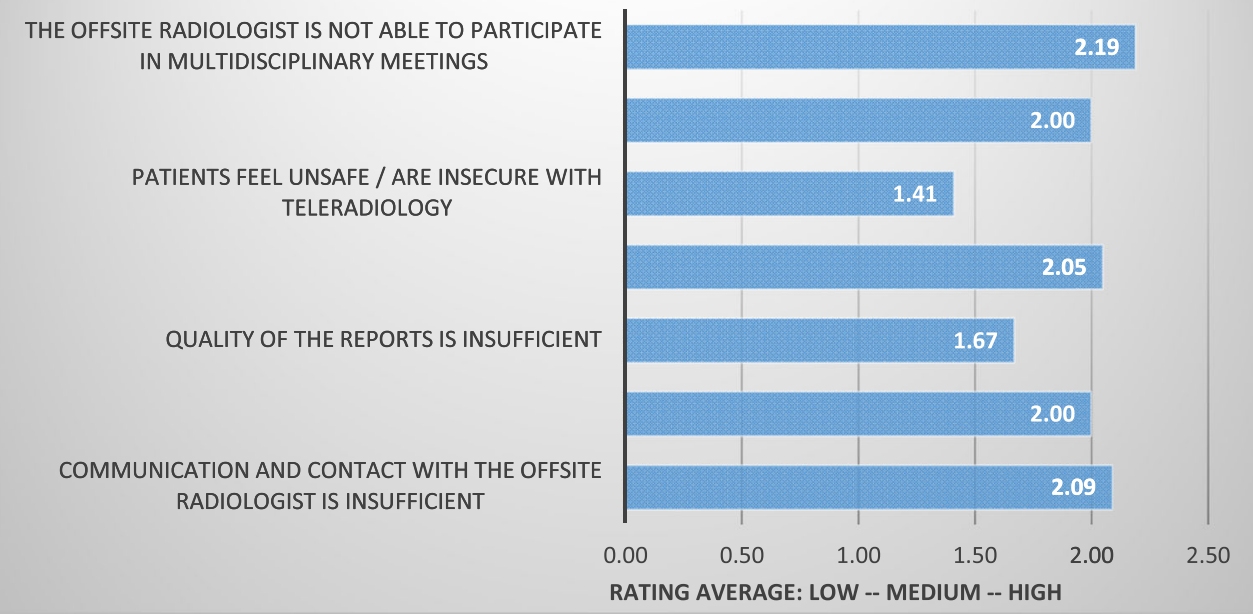

* score in each field requested 


\section{Are teleradiology services reimbursed in your country?}

Teleradiology is not reimbursed, the hospital pays for it

$53,6 \%$

I don't know

Teleradiology is reimbursed by a national or private health insurance (e.g. NHS)

Teleradiology is not reimbursed, the radiologists pay for it

Teleradiology is only reimbursed for ambulatory care

Teleradiology is only reimbursed for scientific projects

$0,3 \%$

\section{How are price agreements made for provision of teleradiology services?}

The price negotiations are done by the hospital management

$45,7 \%$

I don't know

$25,7 \%$

The price negotiations are done by both the management and radiologists

$17,4 \%$

Prices are fixed and non-negotiable in our country

The price negotiations are done by the radiologists

Other (please specify)

$3,0 \%$

\section{How are radiologists paid for offering teleradiology services in your country?}

It enables radiologists to earn additional money privately

$36,2 \%$

Radiologists don't get additional fees for teleradiology, it's included in our employee/hospital salary

I don't know

It enables radiologists to increase their hospital salary

Teleradiology services are added to the total revenue/budget of the radiology department

$30,3 \%$

$19,4 \%$

$11,8 \%$

Teleradiology services are used to fund a separate budget for the radiology team

$8,2 \%$

Other (please specify)

\section{Competition: is teleradiology in your country causing a price competition?}

Competition: is teleradiology in your country causing a price competition?

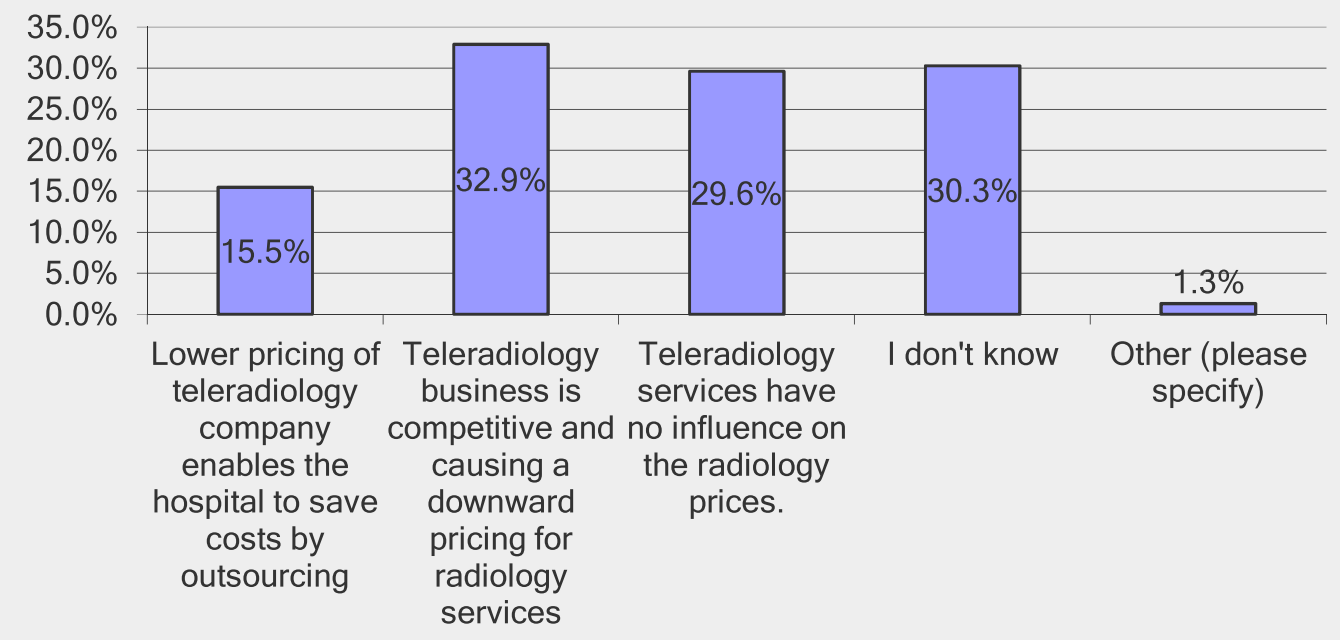


23. Do you believe that teleradiology is a threat for private / hospital based radiologists in your country?

No

$34,9 \%$

Yes, teleradiology is devaluating our profession to a "commodity" that can be replaced

The hospital still prefers local radiologists for better services, although they could make savings with outsourcing

Yes, hospital managers are threatening with outsourcing and increasing pressure on local radiologists

Yes, commercial / private teleradiology providers are even trying to replace local radiologists (predatory business)

Yes, young radiologists will find fewer jobs in hospitals

$27,6 \%$

I don't know

Other (please specify)

\section{Are patients informed that you are using teleradiology for outsourcing?}

No, patients don't know this at all

$52,2 \%$

I don't know

$15,1 \%$

Yes, patients are informed before the examination but don't need to give their approval

$12,0 \%$

No, but patients know this because it's common practice

$8,0 \%$

Yes, patients are informed before the examination and need to give their approval

(informed consent)

$8,0 \%$

Other (please specify)

$4,7 \%$

\section{Can patients refuse that their images are "outsourced" for teleradiology?}

\section{Can patients refuse that their images are "outsourced" for} teleradiology?

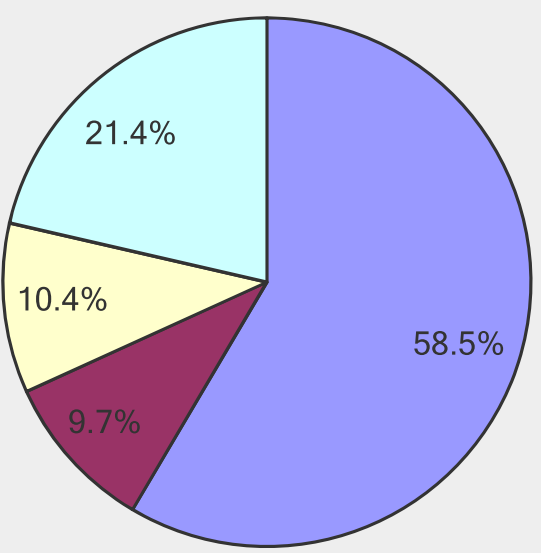

$\square$ No, they don't know that their images are being outsourced

Yes they are able to refuse it and to chose another solution

口Yes they are able to refuse it but then there's no alternative

미 don't know 
26. Are patients satisfied with teleradiology in your working environment?

Are patients satisfied with teleradiology in your working environment?

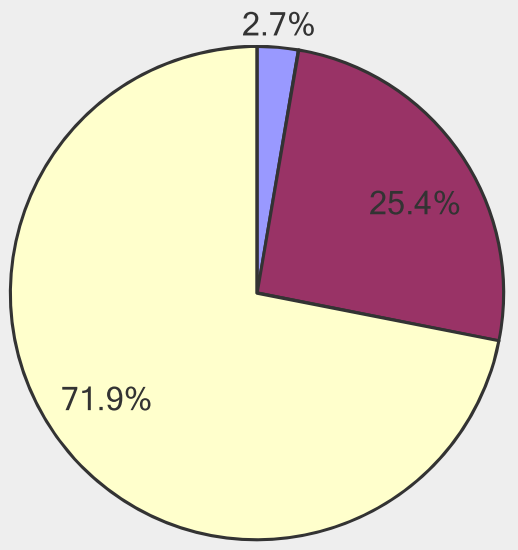

$\square$ No

aYes

I don't know

\section{Did patients ever refuse the usage of teleradiology?}

\section{Did patients ever refuse the usage of teleradiology?}

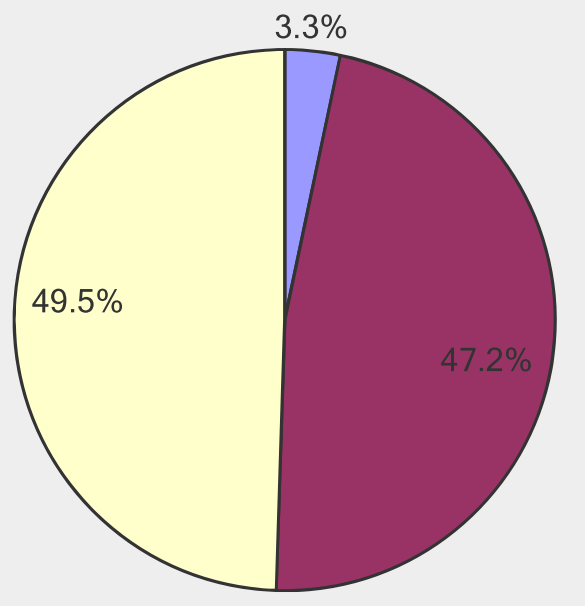

口Yes

$\square$ No

미 don't know

28. Does a non-radiologist have to interpret the images in situations where no radiologist is available in your working environment (neither onsite nor offsite)?

We don't have that kind of situation

$59,9 \%$

The referring doctor reads the images, the final read is made by a radiologist in a $25,8 \%$ 
later stage

Other (please specify)

The referring doctor reads the images, no radiology report is made $\quad 5,0 \%$

A trained radiographer does a preliminary read

\section{Do you have knowledge of any existing regulation, legislation or guidelines about} teleradiology (both on a national and European level)?

I don't know about any existing legislation, regulation or guidelines

I know about the existence of national rules

I know about the existence of national and European guidelines (ESR) and legislation

(EU)

I know about the existence of ESR guidelines (white paper)

I know about the existence of ESR guidelines and European legislation

I know about the existence of European legislation
$44,5 \%$
$20,4 \%$
$15,4 \%$
$13,2 \%$
$3,5 \%$
$3,0 \%$

30. Are you working with cross-border / international teleradiology for FINAL readings (not preliminary, not expert opinions)?

No, all offsite radiologists need to be located in the country where the examination is performed

$55,3 \%$

I don't know

$17,8 \%$

Images can be read from another country by a radiologist registered in the country of examination

Only for expert opinions / research / scientific purposes

$10,0 \%$

$7,8 \%$

Images can be read from another country by a radiologist registered in any EU member state

Images can be read from another country by a radiologist registered in a non-EU member state
$6,7 \%$

$2,4 \%$

31. The offsite radiologist does not need to register in the patient's country if he/she lives in a EU member state, as long he/she doesn't physically move to that country to work as radiologist (European Directive 2011/24 on the mobility of patients' rights). Local registration and accreditation are only necessary for radiologists from outside the EU. What do you think about this?

The offsite radiologist does not need to register in the patient's country if he/she lives in a EU member state, as long he/

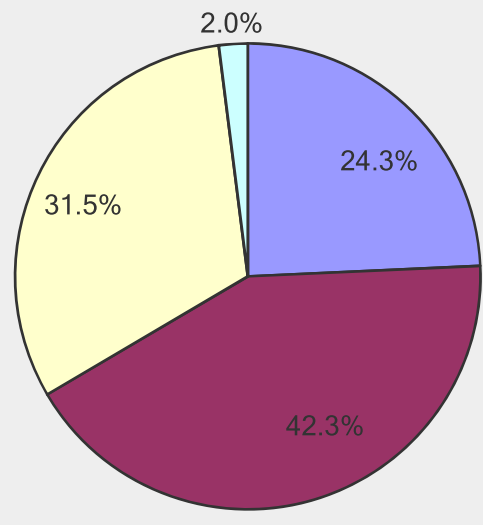

口EU legislation regarding international teleradiology in Europe (EU) is clear and has to be applied

-Radiologists should still register in the country of the patient, also radiologists from within the EU

미 don't know

口Other (please specify) 


\section{Discussion}

This second survey sent to ESR members is more extensive and covers a wide range of topics and issues. A summary of the specific answers to the questions regarding these different topics is listed below.

\section{Usage of teleradiology (TR)}

The majority of the respondents (68.3\%) answered positively to the use of teleradiology (in- or outsourcing), whereas one third $(31.7 \%)$ answered that they did not use teleradiology (in- or outsourcing).

\section{Outsourcing}

For outsourcing, $51.7 \%$ stated that they did not use 'TR outsourcing', whereas $27.6 \%$ indicated that 'readings are outsourced from their backlog', $23.4 \%$ answered that 'on-call readings are outsourced during nights and weekends' and $6.8 \%$ stated that 'specialised readings are outsourced due to lack of experience'.

Almost half of the respondents (49.2\%) stated that both management and radiologists are involved in the decisionmaking process about outsourcing. One third (30.6\%) stated that solely the management decides on outsourcing. In addition, $13.7 \%$ stated that the radiologists decide by themselves about when and how to outsource and about $4 \%$ did not know about the decision-making process.

\section{Insourcing}

More than one third (32.5\%) of the respondents stated that their organisation uses TR insourcing, whereas $25.6 \%$ stated it was used for 'expert/subspecialty readings', $21.5 \%$ stated it was used for on-call purposes, and $13.8 \%$ stated that work lists are being shared between private hospitals within the same hospital group. Almost a quarter (23.4\%) of the respondents stated that they do not use insourcing, and $8.5 \%$ stated that they do not use in- or outsourcing at all, while $3.6 \%$ stated that they did not know about the procedure.

\section{Other purposes}

There were $41.6 \%$ that stated TR is not used for any other purposes, whereas $40.2 \%$ responded that TR is used for providing expert opinions (business-to-business); $15.4 \%$ stated that TR is used for teaching purposes, $12.1 \%$ for scientific research, and $6,1 \%$ for providing expert opinion directly to the patient. In addition, $8.3 \%$ do not know about other purposes, and $0.6 \%$ do not use TR at all.

\section{Location}

For location, $44.1 \%$ of the respondents stated that TR is used on a workstation in their hospital/department; $14.8 \%$ stated that TR is used on a workstation located in the office of the TR company, $14.5 \%$ responded that they use a combination of workstation, mobile device and own PC/laptop, and $1.2 \%$ stated that they use a hospital-owned mobile device. Also, $12.7 \%$ use TR at home on a professional workstation with equivalent performance to hospital PACS, $11.5 \%$ use TR at home on their own computer, and $1.2 \%$ use other private mobile devices (smartphone, tablet).

\section{Communication}

The vast majority of respondents $(78.9 \%)$ stated that TR reports are automatically incorporated in their PACS/RIS systems, whereas $17.8 \%$ stated that the offsite radiologists communicate by phone, normal email, or other online technique (VOIP). Also, $12.4 \%$ stated that offsite radiologists communicate via a secured e-mail system (e.g., DICOM-e-mail protocol adopted as a national standard in Germany or other third party secure Email systems) and $8.8 \%$ stated that the offsite radiologist sends a report by fax, while $4.5 \%$ stated that they did not know about the communication protocols used and $0.3 \%$ responded that the offsite radiologists sends an SMS.

Additionally, $53.8 \%$ of the respondents stated that the offsite radiologist can be contacted by phone on a $24 / 7$ basis, and $10.6 \%$ stated that the offsite radiologist can participate in multidisciplinary meetings from a distance using a secure online platform, whereas $9.1 \%$ stated that the offsite radiologist brings visits to their hospital on a regular basis, and 7.9\% stated that the offsite radiologist is available for video-consultations, e.g., via Skype, GTM, or other software. Also, 16.3\% responded that there is no possibility to discuss the results with the offsite radiologist at all and $15.7 \%$ do not know.

Furthermore, $64 \%$ of the respondents stated that the referring clinician can contact the offsite radiologist, whereas $49.8 \%$ stated that the radiographer contacts the offsite radiologist, and $48.3 \%$ stated that local (onsite) radiologist contacts the offsite radiologist. According to $21.1 \%$ of the respondents, the management contacts the offsite radiologist, and $13.3 \%$ do not know about the procedure. Also, $2.1 \%$ stated that the patient can directly contact the offsite radiologist.

\section{Accessibility and quality assurance}

For accessibility and quality assurance, $48.9 \%$ of the respondents stated that the offsite radiologist has access to the 
PACS/RIS and, thus, is able to view prior studies and results, and $17.8 \%$ stated that the offsite radiologist has access to all relevant patient information including electronic patient records and PACS/RIS. Also, $15.7 \%$ responded that the offsite radiologist does not have access to PACS/RIS and prior studies cannot be viewed from elsewhere, and $7.6 \%$ stated they do not know if offsite radiologists have access to prior imaging results and/or patient information.

Almost half (48.9\%) of the respondents stated that their organisation uses a quality assurance system for TR, whereas $32.6 \%$ stated that they do not use a quality assurance system in their organisation for TR, and $20.6 \%$ do not know whether their organisation uses quality assurance systems for TR.

Then $36.3 \%$ of the respondents stated that they do not know what type of quality assurance systems are being used by their organisation for TR, whereas $26.8 \%$ stated that offsite radiologists need to be registered in their country and need to follow the national guidelines for local accreditation, $16.3 \%$ stated that double readings are made before sending the report and $14.5 \%$ stated that random double readings are made by local radiologists. In addition, $13.5 \%$ of the respondents stated that the offsite radiologist or teleradiology company needs formal approval of the medical staff/directors and $12.9 \%$ stated that in their organisation the TR services are being audited on a regular basis and $9.8 \%$ indicated other.

\section{Satisfaction, advantages \& disadvantages of TR}

More than two thirds $(62.7 \%)$ of the respondents stated that they think that the referring doctors are satisfied with the TR services within their working environment, whereas $18.5 \%$ stated that they did not know and $18.8 \%$ stated that they believe that the referring doctors are unsatisfied with the TR services.

The majority of respondents rated 'greater availability of radiologists' as the most important advantage of $\mathrm{TR}$, second is 'faster turnaround time, clinicians get faster results', 'more easily availability of subspecialty knowledge' is in third place followed by 'improvement of the radiologist's lifestyle', 'improvement of local radiologist's workload' and 'the improvement of the overall quality of care'. Whereas 'the improvement of the overall quality of radiology services' seems to be the least accurate advantage to the respondents of the survey.

As regards the disadvantages of TR usage, 'the offsite radiologists unavailability to participate in multidisciplinary meetings' seems to be the most important disadvantage for the respondents of the survey. This is followed by 'insufficient communication and contact with the offsite radiologist' in second place, 'insufficient contact with the patient' in third place and 'the insufficient access of offsite radiologists to clinical and/or historical patient data' and 'insufficient contact between the offsite radiologist and radiographers' in fourth place. 'Insufficient quality of reports' and 'patients feel unsafe / are insecure with TR' mark the least relevant disadvantages according to the respondents.

\section{Reimbursement, pricing, and competition}

More than half $(53.6 \%)$ of the respondents stated that TR is not being reimbursed and that the hospital covers the costs, $23.4 \%$ stated that they do not know whether TR is being reimbursed, $18.8 \%$ stated that TR is reimbursed by a national or private health insurance, only $3 \%$ stated that the radiologists pay for the TR services, $1 \%$ stated that TR is only reimbursed in cases of ambulatory care and $0.3 \%$ stated that TR is only reimbursed for scientific projects.

As regards the pricing arrangements, almost half (45.7\%) of the respondents stated that the hospital management is in charge of price negotiations. A quarter of the respondents $(25.7 \%)$ do not know who is in charge, $17.4 \%$ stated that the negotiations are done by both management and radiologists, whereas $8.2 \%$ stated that prices are fixed and nonnegotiable in their country. Only $4.6 \%$ of the respondents stated that the radiologists are in charge of the price negotiations and $3 \%$ stated 'other'.

According to $36.2 \%$ of the respondents, radiologists earn additional private money through TR, whereas $30.3 \%$ stated that radiologists do not get any additional payments through TR as it is included in the hospital/employee salary. Almost twenty percent (19.4\%) stated that they do not know how TR services are being paid for. More than ten percent $(11.8 \%)$ stated that TR increases their hospital salaries and $8.2 \%$ stated that TR services are added to the total revenue/budget of the radiology dept. Only $3.9 \%$ stated that TR services are used to fund a separate budget for the radiology team.

As regards the potential impact of TR on price competition, $32.9 \%$ of the respondents stated that 'TR is competitive and causing a downward pricing for radiology services', whereas $29.6 \%$ stated that 'TR does not influence radiology prices' and $30.3 \%$ did not know about potential impacts on price competition. Also, $15.5 \%$ stated that 'lower pricing of TR companies enable hospitals to save costs via outsourcing', and 1.3 stated 'other'.

\section{Potential threat by TR for radiologists}

Almost thirty-five percent (34.9\%) of the respondents stated that TR is no threat for private/hospital based radiologists, whereas $30.9 \%$ stated that 'TR devaluates the radiology profession to a "commodity" that can be replaced' and $20.7 \%$ stated that 'hospital managers are threatening radiologists with outsourcing and increasing the pressure on local radiologists'. In addition, $18.8 \%$ stated that 'commercial/private TR providers are even trying to replace local radiologists' and $13.8 \%$ fear that 'younger radiologists will find fewer jobs in hospitals 
through TR services'. Almost one third (27.6 \%) believe that 'hospitals still prefer local radiologists for better services, although they could make savings with outsourcing' and 7.6\% do not know if TR is a possible threat to local onsite radiologists.

\section{Patient information and satisfaction}

More than half $(52.2 \%)$ of the respondents stated that 'patients do not know at all that TR is being used for outsourcing' and $8 \%$ stated that 'the patients do not know that TR is being used for outsourcing, but the patients are aware that it is common practice to do so', whereas $12 \%$ stated that 'patients are informed that TR services is being used for outsourcing but no approval by the patient is needed' and $8 \%$ stated that 'informed consent by the patient is needed to use TR for outsourcing', and $4.7 \%$ stated 'other'.

The vast majority of respondents $(71.9 \%)$ stated that they do not know if patients are satisfied with TR in their working environment, whereas $25.4 \%$ stated that they believe that the patients are satisfied with TR and $2.7 \%$ stated that they do not believe that patients are satisfied with TR.

Almost half (49.5\%) of the respondents stated that they do not know any cases where the patient refused TR, whereas $47.2 \%$ stated that they never had the situation of patients refusing TR, and $3.3 \%$ stated that they had the case where patients refused TR.

\section{TR readings by non-radiologists}

Almost sixty percent $(59.9 \%)$ of the respondents stated that they never experienced the situation of a 'non-radiologist' having to interpret the images due to unavailability of onsite radiologists, whereas $25.8 \%$ responded that the referring doctors read the images, and the final readings are made by a radiologist in a later stage. Also, $5 \%$ stated that the referring doctors read the images and no radiology report is made and $2.3 \%$ stated that the radiographer does a preliminary read.

\section{Cross-border/international TR and national/EU legislation}

Almost forty-five percent (44.5\%) of the respondents are unaware of any existing legislative framework, regulations or guidelines, and $20.4 \%$ stated that they do not know of any national rules in this regard. While $15.4 \%$ stated that they are aware of the existence of national and European guidelines (ESR) and legislation (EU), whereas $13.2 \%$ stated that they knew about the existence of the ESR guidelines (white paper) and only $3.5 \%$ stated that they knew about ESR guidelines and EU legislation, and only $3 \%$ stated that they knew about the existence of EU legislation.

More than half $(55.3 \%)$ of the respondents stated that all offsite radiologists need to be located in the country where the examination is performed and, thus, did not work with cross-border/international TR for FINAL readings (not preliminary or expert opinions). Also, $17.8 \%$ do not know whether they worked with crossborder/international TR for FINAL readings (not preliminary or expert opinions). In addition, $10 \%$ stated that images can be read from another country by a radiologist registered in the country of examination, $7.8 \%$ stated that only for expert/research/scientific purposes, $6.7 \%$ stated that images can be read from another country by a radiologists registered in any EU member state, and $2.4 \%$ stated that indeed images can be read from another country by radiologists not registered in a non-EU member state.

As regards the EU legislation on the application of patients' rights in the cross-border healthcare (Directive 2011/24/EU) regarding the registration of the offsite radiologist in the patient's country, $42.3 \%$ stated that they believe that the radiologist should still register in the country of the patient including radiologists working within the EU. Whereas $31.5 \%$ responded 'I don't know' and $24.3 \%$ stated that the EU legislation regarding international TR in the European Union is clear to them and needs to be applied, but $2 \%$ responded 'other'.

\section{Conclusions}

The results of this survey confirm a relatively wide adoption of teleradiology in Europe with a variable focus on what is the real application in clinical practice. However, the trend to use cross-border services and outsourcing for remote services remains limited. The lack of general regulations and legislation is of concern and the need for better quality control and some concerns about the level of adequate expertise. Differences between global EU legislation and local and national regulations may result in contradictions that can be confusing. The lack of awareness of the radiology community about these regulations may also be a source of inadequate apprehension of the fundamental guidelines in adopting teleradiology in clinical practice. Unawareness of the quality assurance (QA) methods in place or those that could be adopted was also striking from the fact that $36 \%$ of the users stated that they did not know how QA was performed. It is, however, clear that the general reluctance toward use of teleradiology comes from fear of deteriorating hospital-based radiology and a commoditisation of low 
cost external services. It is also worth mentioning that these surveys showed that in a large majority of cases, patient opinion regarding TR was not known. Perhaps more attention should be paid to the patient's point of view regarding usage of teleradiology, especially in the context of the current patient-centred trend in healthcare.

While the survey provides a wealth of resourceful data, a large range of comments and answers in free text listed in Appendix 1 may require more structured analysis to extract the general trends and opinions.

Acknowledgments This article was kindly prepared by the ESR eHealth and Informatics Subcommittee (Chairman: O. Ratib, members: E. J. Adam, D. Akata, A. Alberich-Bayarri, E. Dion, C. Kahn, E. Kotter, E. Neri, N. Pyatigorskaya, E. Ranschaert, and P. Ross) on behalf of the European Society of Radiology (ESR). It was approved by the ESR Executive Council in February 2016.

\section{Appendix 1}

In order to access the full list of survey answers, please contact the Insights into Imaging editorial office at office@i3journal.org

Open Access This article is distributed under the terms of the Creative Commons Attribution 4.0 International License (http:// creativecommons.org/licenses/by/4.0/), which permits unrestricted use, distribution, and reproduction in any medium, provided you give appropriate credit to the original author(s) and the source, provide a link to the Creative Commons license, and indicate if changes were made.

\section{References}

1. European Society of Radiology (2014) ESR white paper on teleradiology: an update from the teleradiology subgroup. Insights Imaging 5(1):1-8 